`Sección Endocrinología y Diabetes. Hospital Clínico Universidad de Chile. Santiago, Chile.

${ }^{2}$ Unidad de Diabetes. Hospital San Juan de Dios. Santiago, Chile. a Becada del Programa de Título de Diabetes en el Adulto. Hospital Clínico Universidad de Chile. Santiago, Chile. 'Bioquímico.

Sin conflicto de intereses las Dras. M. Rivas, P. Belmar, P. Durruty y G. López.

La Dra. L Sanhueza declara conflicto de intereses con el Laboratorio Novo-Nordisk.

Recibido el 26 de enero de 2016, aceptado el 22 de agosto de 2016.

Correspondencia a: Margarita Rivas M. San Juan de Luz 4186, Depto. 513, Providencia, Santiago. m.margaritarm@gmail.com

\section{Cetoacidosis diabética normoglicémica en el embarazo. Caso clínico}

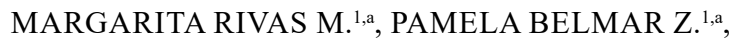 \\ PILAR DURRUTY A. ${ }^{1,2, b}$, LILIAN SANHUEZA M. ${ }^{2}$, GLORIA LÓPEZ S. ${ }^{1}$
}

\begin{abstract}
Normoglycemic diabetic ketoacidosis should be suspected in pregnant women presenting nausea, vomiting, abdominal pain and anorexia. We report a 39 years old woman with a 32 weeks pregnancy who sought emergency care due to hyperemesis. She was hospitalized with the following diagnoses: pregnancy hypertension syndrome, gestational diabetes, morbid obesity and poor prenatal control. The evaluation of the feto-placental unit showed perception of fetal movements, non-reactive non-stress baseline record and a biophysical profile of 6/8. Fetal maturation was initiated. Laboratory tests showed a metabolic acidosis, a low $\mathrm{pH}$, an increased Gap anion, elevated ketonemia and a blood glucose of $172 \mathrm{mg} / \mathrm{dl}$. A diagnosis of normoglycemic diabetic ketoacidosis was formulated and treatment with hydration and regular insulin according to capillary blood glucose levels was started. An emergency caesarean section was performed. The newborn weighed $2.650 \mathrm{~kg}$, had a length of $46 \mathrm{~cm}$, was large for gestational age, had an Apgar score of 2.7, had perinatal asphyxia, convulsive syndrome and a possible congenital cardiopathy. Once the ketoacidosis was resolved during the immediate puerperium, slow acting insulin was initiated.
\end{abstract}

(Rev Med Chile 2016; 144: 1360-1364).

Key words: Diabetes, Gestational; Diabetic Ketoacidosis; Hyperemesis gravidarum.

\section{L} a Cetoacidosis Diabética (CD) en el embarazo, es una emergencia médica que requiere tratamiento en Unidad de Cuidado Intensivo debido a su asociación con mortalidad materna $(5-15 \%)$ y fetal $(9-35 \%)^{1}$. Su frecuencia ha disminuido de 16,0 a $1,2 \%$ gracias a la insulinoterapia y su mejor control metabólico ${ }^{2}$.

La CD se presenta principalmente en pacientes con Diabetes Mellitus tipo 1 (DM1), tipo 2 (DM2) y Diabéticas Gestacionales (DG) ${ }^{3}$.

En el embarazo la CD tiende a ocurrir con niveles bajos de glicemia, cifras $\sim 200 \mathrm{mg} / \mathrm{dl}$ definidas como normoglicémicas ${ }^{4}$. Fue descrita por Munro en $1973^{5}$ y constituyen entre $0,8-1,1 \%$ de las cetoacidosis 6 . La causa exacta se desconoce, hay factores predisponentes: ingesta calórica baja, ayuno prolongado y vómitos excesivos ${ }^{7}$. Algunos autores ${ }^{6}$ ponen en duda la existencia de la Cetoacidosis Diabética Normoglicémica (CDN).

La cetoacidosis afecta a la madre y al feto, la acidosis, la deshidratación, la hiperglicemia, y las arritmias maternas, causan reducción de la perfusión útero placentaria ${ }^{8}$. Los cuerpos cetónicos atraviesan la placenta provocando acidosis, hipoxia ${ }^{9}$ y arritmias fetales que pueden llevarlo a la muerte. A largo plazo la cetoacidosis se asocia a deterioro del desarrollo neurológico ${ }^{10}$.

El tratamiento de CDN se realiza con aporte de volumen y glucosa desde el inicio, insulinoterapia, reposición de electrolitos y monitorización de la madre y del hijo ${ }^{11}$.

Presentamos el caso de una CDN en una 
embarazada en donde el diagnóstico oportuno y tratamiento adecuado puede lograr un resultado exitoso.

\section{Caso clínico}

Se relata en la secuencia cronológica en que se presentó. Servicio de Urgencia Obstétrico del Hospital San Juan de Dios.

Embarazada de 39 años, obesa mórbida (Índice de Masa Corporal 44,8 kg/m²), con $32+2$ semanas de gestación, consulta por presentar hiperémesis de 10 días de evolución; comienzo intermitente, frecuencia e intensidad crecientes hasta 14 episodios/día, escasa ingesta oral, sin dolor abdominal, ni diarrea.

En su primer control obstétrico, a las 14 semanas de gestación, se registra una glicemia de ayunas: $225 \mathrm{mg} / \mathrm{dl}$. En la semana 31 se realizó prueba de tolerancia a la glucosa oral (PTGO): glicemia basal $120 \mathrm{mg} / \mathrm{dl}$ y a los $60 \mathrm{~min} 245 \mathrm{mg} / \mathrm{dl}$. Una matrona del extra sistema, le indicó sólo régimen.

Antecedentes familiares: madre DM2, hipertensa. Personales: no fuma, no bebe alcohol, no usa drogas. No ingiere medicamentos. Obstétricos: un parto, hijo no macrosómico, ningún aborto, no ha presentado DG.

Examen físico de ingreso. Presión arterial de 184/115 mmHg, afebril, Glasgow 15, sin edema, buena perfusión distal, deshidratación leve, con signos de acantosis nigricans. Exámenes cardiopulmonar y abdominal normales. Útero grávido de tono normal con altura $35 \mathrm{~cm}$. Evaluación neurológica: reflejos osteotendinosos normales, sin déficit motor. Glicemia al ingreso $120 \mathrm{mg} / \mathrm{dl}$.

\section{Sala de maternidad}

Hospitalizada con diagnósticos de Síndrome Hipertensivo del Embarazo (SHE), DG, obesidad mórbida y embarazo con mal control prenatal. Plan de tratamiento: monitoreo y control de presión arterial, estudio del SHE y evaluación de la unidad feto placentaria: percepción de movimientos fetales $(+)$, registro basal no estresante (RBNS) no reactivo, perfil biofísico 6/8 (repetido); se realiza maduración pulmonar fetal con una dosis de betametasona $12 \mathrm{mg}$ im.

Evoluciona con cifras tensionales en rangos normales, por lo que se desestima el diagnóstico SHE; pero continúa con vómitos frecuentes.

\section{Unidad de paciente crítico}

El médico internista de turno diagnostica: Diabetes Pregestacional (DPG) con Cetoacidosis Diabética (Tabla 1). Le indica suero fisiológico $(100 \mathrm{ml} / \mathrm{h})$, glicemia capilar cada $30 \mathrm{~min}$, correcciones con insulina regular sc según glicemias: 150-199 mg/dl, 2 U de insulina; 200-249 mg/dl, $4 \mathrm{U} ; 250-299 \mathrm{mg} / \mathrm{dl}, 8 \mathrm{U}$. Los niveles de glucosa capilar fluctuaron entre 145 y $180 \mathrm{mg} / \mathrm{dl}$.

El obstetra sugiere resolución del embarazo a la brevedad; se realiza cesárea de urgencia ( $15 \mathrm{~h}$ después del ingreso).

Características del Recién Nacido (RN): peso $2.650 \mathrm{~kg}$, talla $46 \mathrm{~cm}$, grande para la edad gestacional y Apgar 2,7. Se hospitaliza en Unidad de Cuidado Intensivo Neonatológico por asfixia perinatal, síndrome convulsivo y cardiopatía congénita en estudio.

\section{Unidad de paciente crítico quirúrgico}

En el puerperio inmediato la paciente se encuentra hemodinámicamente estable, disnea leve, Glasgow 15, acidosis metabólica con anión Gap aumentado, cetonemia+++ (Tabla 2). Glicemia $172 \mathrm{mg} / \mathrm{dl}$; se plantea diagnóstico de CDN.

Tratamiento: solución fisiológica $150 \mathrm{ml} / \mathrm{h}$, glucosada $5 \%, 40 \mathrm{ml} / \mathrm{h}$, microinfusor de insulina y aporte de bicarbonato. A las $12 \mathrm{~h}$ evoluciona con criterios de resolución de CD (Tabla 2).

\section{Tabla 1. Exámenes de laboratorio al ingreso en el Servicio de Urgencia Obstétrico}

\begin{tabular}{|ll|}
\hline Glicemia capilar (mg/dl) & 213 \\
Gases en sangre & \\
$\mathrm{pH}$ & 7,15 \\
$\mathrm{HCO}_{3}(\mathrm{mEq} / \mathrm{l})$ & 5 \\
$\mathrm{pCO}_{2}(\mathrm{mmHg})$ & 17 \\
\hline Anión Gap & 22 \\
\hline Cetonemia & +++ \\
\hline Leucocitos & 12000 \\
\hline Proteinuria aislada (g/l) & 0,64 \\
\hline Creatinina (mg/dl) & 0,8 \\
\hline Electrólitos plasmáticos mEq/l NA/K/Cl & $137 / 4,4 / 110$ \\
\hline
\end{tabular}


Tabla 2. Exámenes de laboratorio en la evolución de la cetoacidosis diabética

\begin{tabular}{|c|c|c|c|c|}
\hline & $\begin{array}{c}\text { Sala de } \\
\text { maternidad }\end{array}$ & $\begin{array}{c}\text { Unidad paciente } \\
\text { crítico } \\
\text { Ingreso }\end{array}$ & $\begin{array}{c}\text { Unidad paciente } \\
\text { crítico } \\
6 \mathrm{~h}\end{array}$ & $\begin{array}{c}\text { Unidad paciente } \\
\text { crítico } \\
12 \mathrm{~h}\end{array}$ \\
\hline Hematocrito \% & 41 & 40 & 36 & \\
\hline Hemoglobina (g/dl) & 14 & 14 & 12 & \\
\hline Leucocitos & 12.110 & 19.850 & 14.330 & \\
\hline Plaquetas & 333.000 & 293.000 & 276.000 & \\
\hline Gases en sangre arterial & & 172 & 152 & \\
\hline $\mathrm{pO}_{2} \quad(\mathrm{mmHg})$ & 121 & 109 & 111 & 100 \\
\hline $\mathrm{pCO}_{2}(\mathrm{mmHg})$ & 13 & 10 & 18 & 19 \\
\hline $\mathrm{pH}$ & 7,22 & 7,17 & 7,27 & 7,34 \\
\hline $\mathrm{HCO}_{3}(\mathrm{mEq} / \mathrm{l})$ & 5,3 & 3,6 & 8,3 & 10 \\
\hline Base excess & -20 & -22 & -18 & -13 \\
\hline Cetonemia & +++ & & & \\
\hline Anión Gap & 18 & 20 & 8 & 7 \\
\hline Electrólitos plasmáticos $\mathrm{Na} / \mathrm{K} / \mathrm{Cl}(\mathrm{mEq} / \mathrm{l})$ & $134 / 43 / 109$ & $134 / 4,3 / 110$ & $133 / 4 / 117$ & $135 / 3,5 / 118$ \\
\hline Creatinina (mg/dl) & 0,8 & 0,85 & 0,7 & \\
\hline Pruebas hepáticas & normales & normales & & \\
\hline Ácido láctico (mg/dl) & 17 & 20 & 12 & \\
\hline
\end{tabular}

\section{Sala de maternidad}

Evaluada por equipo de diabetes, se inicia insulina NPH. Posterior al alta, se indica control ambulatorio en la Unidad de Diabetes del Hospital San Juan de Dios.

\section{Discusión}

El caso que se presenta constituye una secuencia de hechos negativos que dan por resultado que la madre tenga una CD en la $32+2$ semanas de embarazo y que el $\mathrm{RN}$ presente varios trastornos.

Los organismos internacionales ${ }^{12,13}$ y nuestro país ${ }^{14}$, consideran que una diabetes diagnosticada en el primer trimestre del embarazo es una DPG.

La paciente en su control obstétrico (14 semanas) presentó hiperglicemia de ayunas, lo que plantearía una DPG y en la PTGO (mal indicada, tenía 31 semanas) cifras elevadas; es discutible el valor de $245 \mathrm{mg} / \mathrm{dl}$ de glicemia realizado a la $1 \mathrm{~h}$ (la norma indica a las $2 \mathrm{~h}$ ), sin embargo, es probable que sería $>140 \mathrm{mg} / \mathrm{dl}$ y la basal estaba fuera de la normalidad, lo que confirma una diabetes no tratada.
El diagnóstico de diabetes en el embarazo se realiza: en el primer trimestre con una glicemia de ayunas repetida $\geq 126 \mathrm{mg} / \mathrm{dl}$, se clasifica como DPG; con los valores entre 100 y $125 \mathrm{mg} / \mathrm{dl} \mathrm{se}$ diagnostica como una DG. En todas las pacientes que se descarta una DPG o DG se les indica una PTGO a las 24-28 semanas; cifras en ayunas $\geq$ $100 \mathrm{mg} / \mathrm{dl} \mathrm{y} / \mathrm{o} 2 \mathrm{~h}$ post carga $\geq 140 \mathrm{mg} / \mathrm{dl}$ son diagnóstico de $\mathrm{DG}^{14}$.

A pesar de la franca hiperglicemia en la semana 14 no se envió al Departamento de Alto Riesgo Obstétrico. Su embarazo evoluciona sin manejo de su condición de diabética y sólo consulta en la semana 32, por presentar hiperémesis. Este error diagnóstico y terapéutico, más la negligencia de la paciente que no realizó seguimiento de su embarazo, la llevó a presentar una CD en el tercer trimestre.

Los cambios fisiológicos del embarazo predisponen a desarrollar $\mathrm{CD}^{15}$; es normal una menor sensibilidad a la insulina, mayor lipolisis y cetogénesis; la concentración de cetonas es dos a cuatro veces superior que en las no embarazadas. Además, la alcalosis respiratoria, disminuye la concentración de bicarbonato sérico, reducien- 
do la capacidad de neutralizar hidrogeniones ${ }^{16}$. Otros factores, especialmente en el segundo y tercer trimestre son: disminución de la ingesta calórica, náuseas, hiperémesis, deshidratación, estrés, aumento de hormonas: glucagón, lactógeno placentario, prolactina y cortisol; los más habituales son las infecciones agudas y la omisión de la insulinoterapia ${ }^{15}$.

La presentación de la CD es similar a la mujer no embarazada: hiperglicemia, cetonemia positiva, alto anión Gap; los niveles de glucosa pueden no ser tan elevados porque la madre y el feto utilizan grandes cantidades de glucosa lo que disminuye en la mujer la glicemia de ayunas ${ }^{17}$.

Con el uso de betametasona durante el embarazo se favorece la hiperglicemia, este efecto se presenta 2 a 4 días después de la administración de 2 dosis de $12 \mathrm{mg}$ del fármaco ${ }^{18}$. En este caso, se inyectó una sola dosis y la resolución del parto se realizó a las $15 \mathrm{~h}$; por lo que no se alcanza a manifestar la acción hiperglicemiante del corticoide; la $\mathrm{CD}$ se manifestó con valores de glicemia cercana a $200 \mathrm{mg} / \mathrm{dl}$.

En esta paciente su embarazo, su diabetes no controlada, los vómitos intermitentes de varios días con baja ingesta calórica, la llevaron a desarrollar una CD: glicemia $213 \mathrm{~g} / \mathrm{dl}$, cetonemia +++ , $\mathrm{pH} 7,15$, bicarbonato muy bajo $(5 \mathrm{mEq} / \mathrm{l})$ y anión Gap elevado, permiten establecer el diagnóstico de CD, la que fue resuelta en forma exitosa en el puerperio inmediato (Tabla 2).

Llama la atención las glicemias moderadas, cercanas a $200 \mathrm{mg} / \mathrm{dl}$; por lo cual esta paciente presentaría una CD normoglicémica. Se ha publicado casos similares en embarazadas, siendo las cifras más bajas de glucosa entre 78-128 mg/dl ${ }^{19,20}$.

En el tratamiento de la CDN se recomienda infusión de glucosa desde el inicio, en este caso se aportó solución glucosada 5\%; además de bicarbonato.

El desencadenamiento de esta complicación aguda va a depender fundamentalmente del mal tratamiento de la diabetes materna. Un factor negativo importante fue la negligencia de la paciente, que sólo consultó varios días después de iniciados los vómitos, ya en plena $\mathrm{CD}$, con el consiguiente efecto deletéreo en el RN.

Los problemas que presentó el hijo, serían por la diabetes no conocida, existiría descompensación desde antes de la concepción. La DPG no tratada de la madre, provocaría daños en el $\mathrm{RN}$ y en la etapa final del embarazo la cetoacidosis sería responsable de los trastornos neurológicos.

Se estima que la CD no es indicación de interrupción inmediata del embarazo existiendo mayor morbilidad si la madre no está estabilizada ${ }^{16,21}$; sin embargo, no hubo problemas por practicar la cesárea cuando la CD no estaba resuelta.

Asi, en las embarazadas diabéticas que presenten vómitos es necesario evaluar el estado ácido base aunque la glicemia sea normal y en las pacientes con DM1 o DM2, el embarazo debe ser programado y con buen control glicémico.

\section{Referencias}

1. Carroll MA, Yeomans ER. Diabetes Ketoacidosis in Pregnancy. Crit Care Med 2005; 33: S347-S353.

2. Schneider MB, Umpierrez GE, Ramsey RD, Mabie WC, Bennett KA. Pregnancy complicated by diabetes ketoacidosis: maternal and fetal outcomes. Diabetes Care 2003; 26: 958-9.

3. Umpierrez GE, Casals MM, Gebhart SP, Mixon PS, Clark WS, Phillips LS. Diabetes Ketoacidosis in obese African-Americans. Diabetes 1995; 44: 790-5.

4. Cullen MT, Reece EA, Homko CJ, Sivan E. The changing presentations of diabetes ketoacidosis during pregnancy. Am J Perinatol 1996; 13: 449-51.

5. Munro JF, Campbell IW, McCuish IW, Duncan LJ. Euglycaemic diabetic ketoacidosis. BMJ 1973; 2: 578-80.

6. Jenkins D, Close CF, Krentz AJ, Nattrass M, Wright AD. Euglycemic diabetic ketoacidosis: does it exist? Acta Diabetol 1993; 30: 251-3.

7. Burge MR, Hardy KJ, Schade DS. Short term fasting is a mechanism for the development of euglycemic ketoacidosis during periods of insulin deficiency. J Clin Endocrinol Metab1993; 76: 1192-8.

8. Blechner JN, Stenger VG, Prystowsky M. Blood flow to the human uterus during maternal metabolic acidosis. Am J Obstet Gynecol 1979; 121: 789-94.

9. Kamalakannan D, Baskar V, Barton DM, Abdu TAM. Diabetic ketoacidosis in pregnancy. Postgrad Med J 2003; 79: 454-7.

10. Rizzo T, Metzger BE, Burns WJ, Burns K. Correlations between ante partum maternal metabolism and child intelligence. N Engl J Med 1991; 325: 911-6.

11. Chauhan SP, Perry KG. Management of diabetic ketoacidosis in the obstetric patient. Obstet Gynecol North Am 1995; 22: 143-55.

12. American Diabetes Association. Standards of Medical Care in Diabetes 2016. Diabetes Care 2016; 39 (Suppl 1): S18. 
13. International Association of Diabetes and Pregnancy Study Groups Recommendations on the Diagnosis and Classification of Hyperglycemia in Pregnancy. Diabetes Care 2010; 33: 676-82.

14. www. Guía Diabetes y Embarazo Minsal 2014.

15. Rodgers BD, Rodgers DE. Clinical variables associated with diabetic ketoacidosis during pregnancy. J Reprod Med 1991; 36: 797-800.

16. de Veciana M. Diabetes ketoacidosis in pregnancy. Semin Perinatol 2013; 37: 267-73.

17. Guo RX, Yang LZ, Li LX, Zhao XP. Diabetic ketoacidosis in pregnancy tends to occur at lower blood glucose levels: case-control study and a case report of euglycemic diabetic ketoacidosis in pregnancy. J Obstet Gynaecol
Res 2008; 34: 324-30.

18. Ramírez-Torres MA, Pérez-Monter SE, Espino y Soza S, Ibargüengoitia-Ochoa F. Efecto de la betametasona en los niveles de glucosa en sangre en mujeres diabéticas embarazadas con riesgo de parto prematuro. Ginecol Obstet Mex 2011; 79: 565-71.

19. Oliver R, Jagadeesan P, Howard RJ, Nikookam K. Euglyceamic diabetic ketoacidosis in pregnancy: An unusual presentation. J Obstet Gynaecol 2007; 27: 308-21.

20. Chico M, Levine SN, Lewis DF. Normoglycemic diabetic ketoacidosis in pregnancy. J Perinatol 2008; 28: 310-2.

21. Chauhan SP, Perry KG. Management of diabetic ketoacidosis in the obstetric patient. Obstet Gynecol North Am 1995; 22: 143-55. 